\title{
Emission and Absorption of Radiation by Spectral Lines with Doppler Contour*
}

\author{
S. S. PENNER \\ Guggenheim Jet Propulsion Center, California Institute of Technology, Pasadena, California
}

(Received October 15, 1951)

\begin{abstract}
Equations are developed for observable intensities in emission and absorption for spectral lines with Doppler contour. It is shown that the theoretical relations which are usually employed in estimating effective temperatures of internal energy states (a) require slight modifications when proper allowance is made for the shape of spectral lines, and (b) are not valid unless the product of spectral absorption coefficient and optical density is sufficiently small. Illustrative calculations on several spectral lines belonging to the $(0,0)$ band of the ${ }^{2} \Sigma \rightarrow{ }^{2} \Pi$ band system of $\mathrm{OH}$ suggest that the conditions under which the basic relations hold are probably not satisfied for representative combustion flames.
\end{abstract}

$I^{T}$ T is well known that the emission of radiation in the visible and ultraviolet regions of the spectrum does not make an important contribution to the total equilibrium flux of radiant energy at the temperatures which are normally encountered in combustion chambers. Radiant energy transfer in these spectral regions is, however, of great importance in spectroscopic studies for estimating population temperatures (i.e., rotational, vibrational, and electronic temperatures). Since most of this work is carried out at atmospheric pressure or even at subatmospheric pressures, as in low pressure combustion flames, it is of obvious interest to examine the intensities of radiation emitted for Doppler-broadened lines. The results are immediately applicable to such problems as emission and absorption intensities of $\mathrm{OH}$, $\mathrm{C}_{2}, \mathrm{CH}$, etc., especially in low pressure flames. For emitters with large optical collision diameters such as sodium, and at moderate and elevated pressures for other chemical species, it is necessary to extend the theory discussed in the present report to spectral lines whose contour is described by combined collision- and Doppler-broadening.

Since one of the important applications of the present discussion is to the interpretation of spectroscopic data obtained in emission and absorption on flames, the following treatment will be presented in such a way as to make the results useful to the spectroscopist. In particular, we shall demonstrate $\nmid$ the following:

(1) For Doppler-broadened lines the maximum intensity of spectral lines observed in emission is proportional to the third power of the frequency rather than to the fourth power of the frequency.

(2) For Doppler-broadened lines the maximum intensity of spectral lines observed in absorption is independent of frequency and not proportional to the frequency.

(3) In absorption experiments particular care must be taken to correct for the spectral emission characteristics of the light source.

We shall allow for the possibility of lack of occurrence of equipartition of energy among the various degrees of

* Supported by the ONR under contract Nonr-220 (03), NR 015210 .

$\dagger$ The treatment given in this report does not allow for instrumental distortions of experimental data. If the instrumental distortions do not depend on frequency, then the conclusions of the present analysis will remain unchanged. freedom by designating the translational (kinetic) temperature by $T_{t}$, the temperature of the upper energy states by $T_{u}$, and the temperature of the lower energy states by $T_{l}$. A further generalization to represent the temperatures of internal degrees of freedom as a function of energy level can be introduced without difficulty but will not be considered in the present discussion.

The results of the present analysis indicate that the optical densities of the chemical species $\mathrm{OH}$ are too large, in representative combustion flames, to permit the type of interpretation of experimental measurements which is usually made, unless the reported $f$-values for spectral lines of the ${ }^{2} \Sigma \rightarrow^{2} \Pi$ band system and $(0,0)$ transitions are much too large.

\section{INTENSITIES IN EMISSION}

The integrated absorption $S_{l u}$ of a spectral line resulting from an energy change from the upper energy level $E_{u}$ to the lower energy level $E_{l}$ is given by the wellknown relation ${ }^{1}$

$$
S_{l u}=\left(64 \pi^{4} / 3 c^{3}\right) N_{u}\left(\nu_{l u}\right)^{4}\left(q_{l u}\right)^{2} / c \rho^{0}\left(\nu_{l u}\right),
$$

where $c=$ velocity of light; $N_{u}=$ number of molecules per unit volume per unit pressure in the upper energy state; $\nu_{l u}=$ frequency of the emitted or absorbed radiation at the center of the line, which is obtained from the Bohr frequency relation; $q_{l u}=$ matrix element corresponding to transitions between the two given energy states; and $\rho^{0}\left(\nu_{l u}\right)=$ volume density of blackbody radiation at the frequency $\nu_{l u}$ as given by the Planck distribution law.

The integrated intensity of a single emitted spectral line is obtained from the relation ${ }^{1}$

$$
\int_{\Delta \nu_{l u}} R_{\nu} d \nu=\frac{1}{4} \int_{\Delta \nu l_{u}} c \rho^{0}(\nu)\left[1-\exp \left(-P_{\nu} X\right)\right] d \nu,
$$

where $\Delta \nu_{l u}$ is a suitable spectral range including the spectral line under study, $R_{\nu}=$ spectral intensity of the emitted radiation, $P_{\nu}=$ spectral absorption coefficient, $X=$ optical density of the emitters. For the Dopplerbroadened spectral line whose center lies at the fre-

${ }^{1}$ For the origin of this relation see, for example, A. C. G. Mitchell and M. W. Zemansky, Resonance Radiation and Excited Atoms (Cambridge University Press, London, 1934). 
quency $\nu_{l u}$ the spectral absorption coefficient $P_{\nu}$ is

$$
\begin{aligned}
\left.P_{\nu}=S_{l u}\left(m c^{2} / 2 \pi k T_{t} \nu_{l u}\right)^{2}\right)^{\frac{1}{3}} & \\
& \times \exp \left[-\left(m c^{2} / 2 k T_{t} \nu_{l u}{ }^{2}\right)\left(\nu-\nu_{l u}\right)^{2}\right],
\end{aligned}
$$

where $m$ represents the mass per molecule.

The optical density $X$ of emitters used in combustion spectroscopy for determining rotational, vibrational, or electronic temperatures may be so small that it is permissible to expand the exponential in Eq. (2) retaining only the first two terms. ${ }^{2}$ Furthermore, the range of frequencies for which $P_{\nu} X$ is significantly different from zero is so narrow that $\rho^{0}(\nu)$ may be replaced by $\rho^{0}\left(\nu_{l u}\right)$. Thus

$$
\int_{\Delta v_{l u}} R_{\nu} d \nu=\frac{1}{4} c \rho^{0}\left(\nu_{l u}\right) X \int_{\Delta \nu l} P_{\nu} d \nu=\frac{1}{4} c \rho^{0}\left(\nu_{l u}\right) S_{l u} X
$$

Comparison of Eqs. (1) and (4) leads to the conclusion that

$$
\begin{aligned}
\int_{\Delta v_{l u}} R_{\nu} d \nu /\left(\nu_{l u}\right)^{4} g_{u}\left(q_{l u}\right)^{2} & \\
& =\left(16 \pi^{4} N X / 3 c^{3} Q\right) \exp \left(-\dot{E_{u}} / k T_{u}\right)
\end{aligned}
$$

where $N_{u}$ has been replaced by

$$
N_{u}=N g_{u} \exp \left(-E_{u} / k T_{u}\right) / Q,
$$

with $N=$ total number of molecules per unit volume per unit pressure, $g_{u}=$ statistical weight of the upper energy state, $Q=$ complete partition function. The optical density $X$ appearing in Eq. (5) refers to all of the emitting molecules independently of the energy state in which they happen to be. Hence we obtain from Eq. (5) the following useful result

$$
\frac{\partial \ln \left[\int_{\Delta \nu l u} R_{\nu} d \nu /\left(\nu_{l u}\right)^{4} g_{u}\left(q_{l u}\right)^{2}\right]}{\partial E_{u}}=-\frac{1}{k T_{u}} .
$$

Equation (7) is the relation which is usually employed for the determination of temperatures from emission spectra for various internal degrees of freedom.

In practice it is convenient to measure $\left(R_{v}\right)_{\max }$, i.e., the maximum intensity of the various spectral lines, rather than the integrated intensity. However, in this case Eq. (7) does not apply exactly since allowance must be made for the frequency dependence of the Dopplerwidth of spectral lines. From Eq. (3) it is apparent that the maximum value of the spectral absorption coeffcient, corresponding to the maximum value of $R_{v}$, is

$$
\left(P_{v}\right)_{\max }=S_{l u}\left(m c^{2} / 2 \pi k T_{t}\right)^{\frac{1}{2}}\left(1 / \nu_{l u}\right) \text {. }
$$

Hence

$$
\left(R_{v}\right)_{\max }=\frac{1}{4} c \rho^{0}\left(\nu_{l u}\right) S_{l u} X\left(m c^{2} / 2 \pi k T_{t}\right)^{\frac{1}{2}\left(1 / \nu_{l u}\right)}
$$

2 This approximation is examined critically for OH in Sec. III. and the relation corresponding to Eq. (5) is

$$
\begin{aligned}
& \left(R_{\nu}\right)_{\max } /\left(\nu_{l u}\right)^{3} g_{u}\left(q_{l u}\right)^{2} \\
& \quad=\left(16 \pi^{4} N X / 3 c^{3} Q\right)\left(m c^{2} / 2 \pi k T_{t}\right)^{\frac{1}{2}} \exp \left(-E_{u} / k T_{u}\right) .
\end{aligned}
$$

Thus

$$
\frac{\partial \ln \left[\left(R_{\nu}\right)_{\max } /\left(\nu_{l u}\right)^{3} g_{u}\left(q_{l u}\right)^{2}\right]}{\partial E_{u}}=-\frac{1}{k T_{u}},
$$

and the factor $\left(\nu_{l u}\right)^{3}$ is seen to replace the factor $\left(\nu_{l u}\right)^{4}$ which is normally employed for Doppler-broadened spectral lines. It should be noted that implicit in Eq. (11) is the assumption that the translational temperature, which determines the Doppler-width of spectral lines, is uniform and, in particular, is independent of $\nu_{l u}$.

\section{INTENSITIES IN ABSORPTION}

In an absorption experiment the integrated intensity of the transmitted radiation is given by the relation ${ }^{1}$

$$
\int_{\Delta \nu l u} R_{\nu} d \nu=\int_{\Delta v l u} R_{0 v}\left[\exp \left(-P_{\nu} X\right)\right] d \nu,
$$

where $R_{0}$ represents the spectral intensity of the incident radiation. For the sake of simplicity we consider the case in which the light source may be treated as a greybody emitter in the wavelength range for which measurements are made. Thus

$$
R_{0 \nu}=\frac{1}{4} E_{8} c \rho_{\nu}{ }^{0}\left(T_{8}\right),
$$

where $E_{s}$ is a constant independent of frequency and $T_{s}$ represents the temperature of the source. For absorption measurements on minor component species such as $\mathrm{OH}$, $\mathrm{CH}, \mathrm{C}_{2}$, etc., in flames the assumption is made again that it is sufficient to retain only the first two terms in the expansion of the exponential in Eq. (12). ${ }^{2}$ Thus, for a single spectral line,

$$
\begin{aligned}
\int_{\Delta \nu l u} R_{\nu} d \nu & \simeq \frac{1}{4} E_{s} X c \\
& \times \int_{\Delta v_{l u}} \rho_{\nu}{ }^{0}\left(T_{s}\right) d \nu-\frac{1}{4} E_{s} X c \rho^{0}{ }_{\nu l u}\left(T_{s}\right) S_{l u}
\end{aligned}
$$

but $^{1}$

where

$$
S_{l u}=\left(N_{l} B_{l \rightarrow u}-N_{u} B_{u \rightarrow l}\right) h \nu_{l u} / c
$$

$$
N_{l}=N g_{l}\left[\exp \left(-E_{l} / k T_{l}\right)\right] / Q
$$

represents the number of molecules per unit volume per unit pressure in the lower (initial) energy level $E_{l}$ whose statistical weight is $g_{l}, B_{l \rightarrow u}$ equals the Einstein coefficient of induced absorption, and $B_{u \rightarrow l}$ is the Einstein coefficient of induced emission. Since ${ }^{3}$

$$
g_{l} B_{l \rightarrow u}=B_{u \rightarrow l} g_{u}
$$

See, for example, E. C. Kemble, Fundamental Principles of Quantum Mechanics (McGraw-Hill Book Company, Inc., New York, 1935), p. 450. 
and

$$
B_{u \rightarrow l}=\left(c^{3} / 8 \pi h \nu_{l u^{3}}\right) A_{u \rightarrow l},
$$

where $A_{u \rightarrow l}$ is the Einstein coefficient of spontaneous emission, it follows that ${ }^{1}$

$$
B_{u \rightarrow l} h \nu_{l u}=\left(8 \pi^{3} / 3 h\right) \nu_{l u}\left(q_{l u}\right)^{2} .
$$

Introduction of Eqs. (6), (6a), and (15) into Eq. (14) leads to the result

$$
\begin{array}{r}
S_{l u}=\left(8 \pi^{3} N / 3 h c Q\right) \nu_{l u}\left(q_{l u}\right)^{2} g u\left[\exp \left(-E_{l} / k T_{l}\right)\right] \\
\times\left\{1-\exp \left[\left(-E_{u} / k T_{u}\right)+\left(E_{l} / k T_{l}\right)\right]\right\} .
\end{array}
$$

For representative spectral lines arising from electronic transitions in the visible and ultraviolet regions of the spectrum and for reasonable values of $T_{l}, T_{u}$

$$
\exp \left[\left(-E_{u} / k T_{u}\right)+\left(E / k T_{l}\right)\right] \ll 1,
$$

whence Eq. (16) can be replaced by the following close approximation :

$$
S_{l u}=\left(8 \pi^{3} N / 3 h c Q\right) \nu_{l u}\left(q_{l u}\right)^{2} g u \exp \left(-E_{l} / k T_{l}\right) .
$$

From Eqs. (13) and (17) the following useful relation is

TABLE I. Representative values of integrated absorption for spectral lines of the ${ }^{2} \Sigma \rightarrow^{2} \Pi,(0,0)$ band of $\mathrm{OH}$.

\begin{tabular}{rcc}
\hline & & \\
Line & $\nu_{l u}, \mathrm{~cm}^{-1}$ & $\begin{array}{c}(T / 273.1) S_{l u} \times 10^{-4} \\
\text { cm }^{-2} \text {-atmos }\end{array}$ \\
\hline$Q_{1}(1)$ & $32,474.58$ & 1.64 \\
$(2)$ & $32,458.65$ & 2.06 \\
$(3)$ & 32.441 .90 & 2.28 \\
$(4)$ & $32,423.63$ & 2.46 \\
$(5)$ & $32,403.47$ & 2.55 \\
$(6)$ & $32,380.99$ & 2.64 \\
& & \\
$R_{2}(1)$ & $32,415.51$ & 0.97 \\
$(2)$ & $32,455.70$ & 1.04 \\
$(3)$ & $32,489.49$ & 1.10 \\
\hline
\end{tabular}

obtained:

$$
\begin{aligned}
& \frac{1}{4} E_{s} X C \int_{\Delta \nu l u} \rho_{\nu}{ }^{0}\left(T_{s}\right) d \nu-\int_{\Delta \nu l u} R_{\nu} d \nu \\
&=\left(2 \pi^{3} N E_{s} X / 3 h Q\right) \rho^{0}{ }_{\nu l u}\left(T_{s}\right) \\
& \quad \times \nu_{l u}\left(q_{l u}\right)^{2} g u \exp \left(-E_{l} / k T_{l}\right) .
\end{aligned}
$$

The quantity appearing on the left-hand side of Eq. (18) evidently represents the total integrated absorbed intensity resulting from the energy transition $E_{l} \rightarrow E_{u}$ and will be designated by the symbol $\int_{\Delta \nu_{l u}} A_{\nu} d \nu$. From Eq. (18) it is evident that

$$
\frac{\partial \ln \left\{\left(\int_{\Delta \nu_{l u}} A_{\nu} d \nu\right) / \nu_{l u} g\left(q_{u}\right)^{2} \rho^{0}{ }_{\nu l u}\left(T_{s}\right)\right\}}{\partial E_{l}}=-\frac{1}{k T_{l}} .
$$

Absorption experiments are generally performed with a greybody light source whose temperature $T_{s}$ is ap- preciably greater than the excitation temperature $T_{l}$ of the lower energy levels. Hence $\rho^{0}{ }_{v i u}\left(T_{8}\right)$ will be substantially independent of $E_{l}$ and Eq. (19) reduces to the usual form for estimates of population temperatures on the lower internal energy states. It is, however, evident from Eq. (19) that the spectral distribution of the light source can affect the experimental results in a very significant way if the light source does not possess the assumed spectral radiation characteristics or if the range of values of $E_{l}$ considered covers too wide a frequency range.

If peak intensities, rather than integrated intensities, are measured in absorption, then, proceeding as in Sec. II, Eq. (18) should be replaced by the relation

$$
\begin{array}{r}
\left(A_{v}\right)_{\max }=\left(2 \pi^{3} N E_{s} X / 3 h Q\right)\left(m c^{2} / 2 \pi k T_{t}\right)^{\frac{1}{3}} \rho_{\nu l u}\left(T_{s}\right) \\
\times\left(q_{l u}\right)^{2} g u \exp \left(-E_{l} / k T_{l}\right)
\end{array}
$$

and

$$
\frac{\partial \ln \left[\left(A_{v}\right)_{\max } / g\left(q_{l u}\right)^{2} \rho_{v l u}{ }^{0}\left(T_{s}\right)\right]}{\partial E_{l}}=-\frac{1}{k T_{l}} .
$$

Reference to Eq. (21) shows that the factor $v_{l u}$ disappears altogether for Doppler-broadened rotational lines if maximum absorption intensities are measured.

\section{APPLICATION TO EMISSION AND ABSORPTION STUDIES OF REPRESENTATIVE LINES OF OH}

The derivation of Eqs. (7) and (11) was based on the approximations

$$
\begin{aligned}
& 1-\exp \left(-P_{\nu} X\right) \simeq P_{\nu} X \\
& 1-\exp \left[-\left(P_{\nu}\right)_{\max } X\right] \simeq\left(P_{\nu}\right)_{\max } X,
\end{aligned}
$$

respectively. Since $\left(P_{\nu}\right)_{\max } \geqslant P$, we shall examine the range of numerical values $\left(P_{v}\right)_{\max } X$ which is encountered in representative combustion flames.

It is readily shown that the experimentally determined oscillator strengths $f_{l u}$ for transitions between the lower energy level $E_{l}$ and the upper energy level $E_{u}$ are related to $S_{l u}$ according to the expression

$$
S_{l u}=2.3789 \times 10^{7}(273.1 / T) f_{l u},
$$

where $S_{l u}$ is expressed in $\mathrm{cm}^{-2}$-atmos ${ }^{-1}$ at the temperature $T$.

Oscillator strengths for representative spectral lines of $\mathrm{OH}$ for the ${ }^{2} \Sigma \rightarrow \rightarrow^{2} \Pi$ band system for $(0,0)$ transitions have been reported by Oldenberg and Rieke ${ }^{4}$ but should be multiplied by the factor $4.2{ }^{5}$ Representative numerical values of $(T / 273.1) S_{l u}$ for several members of the $Q_{1}$ - and $R_{2}$-branches are summarized in Table I. The numbers in parentheses given after the branch designations refer to the values of $K$ in the notation of Dieke and Crosswhite. ${ }^{6}$ Also listed in Table I are the relevant values of $\nu_{l u}{ }^{6}$ Representative values of $S_{l u}$ and of

4 O. Oldenberg and F. F. Rieke, J. Chem. Phys. 6, 439 (1938).

$\checkmark$ R. J. Dwyer and O. Oldenberg, J. Chem. Phys. 12, 351 (1944).

${ }^{6}$ G. H. Dieke and H. M. Crosswhite, "The ultra violet bands of OH, fundamental data," Bumblebee Series Report No. 87 (November, 1948). 
TABLE II. Numerical values of $S_{l u}$ and $\left(P_{v}\right)_{\max }$ for $Q_{1}(6)$ of the ${ }^{2} \Sigma \rightarrow{ }^{2} \mathrm{M},(0,0)$ band of $\mathrm{OH}$, as a function of temperature.

\begin{tabular}{|c|c|c|}
\hline $\mathrm{T}^{\circ} \mathrm{K}$ & $S_{l \mu} \times 10^{-3}, \mathrm{~cm}^{-2}-\mathrm{atmos}^{-1}$ & $\begin{array}{l}\left(P_{\nu}\right)_{\text {max }} \times 10^{-3} \\
\mathrm{~cm}^{-1} \text { atmos }^{-1}\end{array}$ \\
\hline 1000 & 7.21 & 38.1 \\
\hline 2000 & 3.61 & 13.5 \\
\hline 3000 & 2.40 & 7.32 \\
\hline 4000 & 1.80 & 4.75 \\
\hline 5000 & 1.44 & 3.40 \\
\hline 6000 & 1.20 & 2.59 \\
\hline 7000 & 1.03 & 2.06 \\
\hline 8000 & 0.901 & 1.68 \\
\hline 9000 & 0.801 & 1.41 \\
\hline 10,000 & 0.721 & 1.20 \\
\hline
\end{tabular}

$\left(P_{\nu}\right)_{\max }$ for $Q_{1}(6)$ are listed in Table II as a function of temperature. The numerical values of $\left(P_{v}\right)_{\max }$ were calculated from $S_{l u}$ according to Eq. (8).

Reference to Table II shows that the numerical values of $\left(P_{v}\right)_{\max }$ for the $Q_{1}(6)$ spectral linet are exceedingly large and that, therefore, the approximation of Eq. (23) can be justified only for very small values of the optical density $X$. In representative emission experiments from low pressure combustion flames the partial pressure $p$ of $\mathrm{OH}$ may be of the order of $10^{-3}$ atmos, whereas, the radiation path length $L$ is in the neighborhood of $10 \mathrm{~cm}$. For flames burning at higher pressures, $p$ will increase while $L$ decreases in such a way that $X=p L$ remains nearly constant. Hence it appears that the so-called

$\ddagger$ It is evident from the data listed in Table I that the numerical values for other lines are of the same order of magnitude as the listed data for $Q_{1}$ (see reference 6). "abnormal rotational temperatures," which have been reported by different investigators, ${ }^{7-9}$ may be the result of erroneous interpretation of experimental data unless the experimentally determined oscillator strengths are in error and should really be much smaller than reported by Oldenberg and collaborators. ${ }^{4,5}$ On the other hand, it appears likely that for sufficiently large values of $K$, $S_{l u}$ and $\left(P_{v}\right)_{\max }$ will decrease sufficiently to validate the approximation of Eq. (23). Thus the rotational temperatures obtained from data on large values of $K$ may be significant although the "more normal rotational distribution" of the lines with small values of $K$ cannot be accepted as valid experimental evidence unless the reported $f$-values for spectral lines of $\mathrm{OH}$ are much too large.

An obvious extension of the preceding discussion to rotational temperatures determined from absorption experiments would appear to raise serious objection to the so called "normal rotational temperatures of $\mathrm{OH}^{\text {" }}$ reported from absorption measurements. However, in this case the available experimental evidence on the measurement of rotational temperatures ${ }^{9}$ seems so convincing that a serious reexamination of $f$-values appears to be indicated. ${ }^{10}$

${ }^{7}$ A. G. Gaydon and H. G. Wolfhard, Proc. Roy. Soc. (London) 194A, 169 (1948), and later publications.

Penner, Gilbert, and Weber, J. Chem. Phys. 20, 522 (1952).

- H. P. Broida, in National Bureau of Standards Report No. 1123 (August 20, 1951).

${ }_{10}$ Theoretical calculations of $f$-values for $\mathrm{OH}$ have been carried out by R. S. Mulliken, J. Chem. Phys. 8, 382 (1940). 